\title{
La competencia desarrollar sistemas web en la formación de los profesionales informáticos: una aproximación a su estudio
}

\author{
Luis Antonio Llerena Ocaña. Universidad Autónoma de los Andes (UNIANDES) \\ Walfredo González Hernández. Universidad de Matanzas.
}

\section{Recepción: 14 de abril de 2017 | Aceptado: 30 junio de 2017 \\ Correspondencia: Luis Ocaña| Correo-e: Ileroc1@gmail.com}

\section{iD http:// orcid.org/0000-0001-6440-0167}

Citar: Llerena Ocaña, LA. y González Hernández, W. (2017). La competencia desarrollar sistemas web en la formación de los profesionales informáticos: una aproximación a su estudio. ReiDoCrea, 6, 229-245.

\begin{abstract}
Resumen: Las competencias profesionales es uno de los temas más polémicos en el orden psicológico y didáctico pues constituyen el enlace fundamental entre la formación universitaria y las organizaciones empleadoras. En este artículo se aborda una de las competencias del profesional informático que más necesita en los procesos de informatización actual: el desarrollo de sistemas web. El primer acápite se dedica a la actividad de desarrollo de estos sistemas para caracterizarla, en el segundo se devela la estructura de la competencia y se define.
\end{abstract}

Palabras clave: Competencia desarrollo de sistemas web | Enseñanza de la informática

An approach to the study of competence in web systems development in the training of computer professionals

\begin{abstract}
Professional skills are one of the most controversial issues in the psychological and didactic order as they constitute the fundamental link between university training and employing organizations. This article addresses one of the competencies of the computer professional most needed in the current computerization process: the development of web systems. The first section is devoted to the development activity of these systems to characterize it, while in the second, the structure of the competency is unveiled presented and defined.
\end{abstract}

Keywords: Competence in web systems development | Computer education

\section{Introducción}

Con la llegada de Internet muchas formas de comunicación cambiaron de una manera acelerada y la difusión de los conocimientos su alcance a todos se realiza de una manera fácil. De ahí nació la World Wide WEB (más conocida como Web) y las páginas web estáticas inundaron a internet por lo que había muy poca interactividad. Posteriormente se comenzaron a implementar los primeros elementos de interactividad entre la web y los usuarios, llegando a conformar aplicaciones web que gestionaban datos y brindaban información al incorporar bases de datos. A diferencia de las primeras páginas web, las aplicaciones ya permitían acceder a determinados datos, consolidar información, determinar tendencias y, por supuesto, lograr mayor interactividad con los usuarios. Sin embargo, aún no había terminado el proceso puesto que estas aplicaciones se encontraban aisladas y no compartían información entre ellas. Con el advenimiento de los sistemas web el intercambio de información se daba entre aplicaciones y podía ser que una información solicitada por el usuario se encontrara en otra aplicación. También otra situación se encontraba en que una de estas aplicaciones necesitaba datos de otra para funcionar. De esta manera se fue construyendo el entramado de sistemas web tal y como hoy se conoce. La web 2.0 y 3.0 se sustentan en la integración funcional de estos sistemas web. 
Para el desarrollo de estos sistemas intervienen muchos profesionales entre los cuales se destaca el informático. Este profesional es uno de los encargados de desarrollar estos sistemas web y en ello debe integrar conocimientos y habilidades de muchas disciplinas de su formación, entre ellas las redes, ingeniería del software, programación, entre otras que dependen de las características del sistema. Es conocido, además, en la literatura al respecto, que debe integrar un equipo de desarrollo en el cual puede ocupar diferentes roles (Silió, 2005; Williams \& Figueiredo, 2014). Si a ello se le suma la necesidad de una conducta ética respecto a la información de los datos de los usuarios y las organizaciones que gestionan, entonces se está describiendo los modos de actuación de este profesional. Otra arista de la formación de este profesional está en la rápida obsolescencia de los contenidos universitarios

Sin embargo, cuando analizan las estadísticas de pérdidas por conceptos de sistemas web mal desarrollados (Grillo, Cardinale, \& Aguilar, 2015) se demuestra que existen grandes pérdidas por concepto de sistemas web fallidos y se debe fundamentalmente a errores en el proceso de desarrollo. Por ende se evidencian fallas en su proceso de formación como profesionales informáticos.

La búsqueda de referentes acerca del proceso de formación de estos profesionales en la literatura actual (Castañeda, 2017; Corral \& Fernández, 2017) arrojó el análisis de varias competencias relacionadas con la informática, sin embargo, no se aprecia referente en cuanto al desarrollo de aplicaciones web. En otros autores (Dey et al., 2016; Jain \& Suman, 2015; Sudhaman \& Thangavel, 2015) se hace referencia al análisis de requerimientos como proceso fundamental para un correcto desarrollo de aplicaciones sin considerar el tipo de plataforma a la que se le oriente. De ahí que las competencias serían cada uno de las fases de desarrollo genérico de un software declaradas en la literatura al respecto (Pressman, 2011; Pressman \& Lowe, 2013)

Todos estos argumentos refuerzan la necesidad de demostrar que el desarrollo de sistemas web es una competencia, así como develar su estructura interna a partir de un análisis teórico de los referentes acerca de las competencias, sus diferentes clasificaciones y sus componentes.

Los estudiantes universitarios de la carrera de Ingeniería en Computación e informática de la Universidad Regional Autónoma de los Andes de Ecuador y la Carrera de Ingeniería Informática de la Universidad de Matanzas, manifiestan insuficiencias en su formación, relacionadas con el desarrollo de los sistemas web que se imparten en los programas del proceso docente como modo de actuación profesional, lo cual limita la calidad de su labor profesional en los centros de inserción laboral.

Según los resultados de las encuestas aplicadas del Encuentro Nacional de Graduados de la Carrera de Sistemas de la Universidad Regional Autónoma de Andes realizado en los años 2014 y 2015, se ha logrado constatar que el graduado tiene problemas durante la inserción laboral haciendo hincapié en el hecho que los conocimientos adquiridos en el desarrollo de aplicaciones web son muy limitados y se hace mención a que dichos procesos o procedimientos no aprendidos en clase son los principales problemas. En revisiones realizadas a los Trabajos de Diploma de la Carrera de Ingeniería Informática de la Universidad de Matanzas se detectan insuficiencias tales como un diseño poco usable y accesible, brechas de seguridad, pobre uso de lenguajes de programación para este tipo de sistemas 
Estudiar este problema es de gran actualidad porque la praxis histórico social del presente siglo exige del egresado un conocimiento más globalizado de los fenómenos que se dan en el proceso pedagógico; reconceptualizar entonces la concepción del modo de actuación del profesional de la carrera de Ingeniería en computación e informática, adecuándolo a la sociedad del conocimiento, permitirá que la dirección de este proceso sea más acertada, pues contribuirá a conformar en los estudiantes un sistema de competencias que lo acerquen de manera sistémica e integradora al modelo del profesional a que se aspira.

\section{Desarrollo}

\section{1- Sistemas web: surgimiento y tendencias actuales}

Según Luján Mora (2010), "En la ingeniería de software se denomina aplicación web a aquellas aplicaciones que los usuarios pueden utilizar accediendo a un servidor web a través de Internet o de una intranet mediante un navegador. En otras palabras, es una aplicación software que se codifica en un lenguaje soportado por los navegadores web en la que se confía la ejecución al navegador". Este autor hace énfasis al plantear que las aplicaciones web son populares debido a lo práctico del navegador web como cliente ligero relativo a la independencia del sistema operativo, así como a la facilidad para actualizar y mantener aplicaciones web sin distribuir e instalar software a miles de usuarios potenciales. Existen aplicaciones como los web mails, wikis, weblogs, tiendas en línea y la propia Wikipedia que son ejemplos bien conocidos de aplicaciones web.

Es importante mencionar que una página Web puede contener elementos que permiten una comunicación activa entre el usuario y la información. Esto permite que el usuario acceda a los datos de modo interactivo, gracias a que la página responderá a cada una de sus acciones, como por ejemplo llenar y enviar formularios, participar en juegos y acceder a gestores dé base de datos de todo tipo.

No es novedad que el desarrollo de las aplicaciones web vaya evolucionando en el mundo empresarial y su forma de hacer negocios por todo el mundo, sin embargo, para poder llevar a cabo estos grandes avances es necesario incluso en la actualidad ir creando nuevas tecnologías. Pero esta evolución de tecnología es particular de cada empresa, lo que produce una incompatibilidad entre tecnologías de cada empresa.

Señala además que para esto se crearon compañías encargadas de la creación de nuevas especificaciones o estándares, en las cuales, las empresas creadoras de tecnologías deben basarse. Según Andreu, K. (2011) "Una empresa pionera en la creación de estos estándares W3C (World Wide Web)". Cabe recalcar que estos son modeladores de datos, los cuales hacen que la interfaz gráfica en la que el usuario está trabajando sea cómoda, amigable y flexible a cualquier entorno o pantalla en la que se muestre, a esto la W3C le conoce como responsive desing (diseño adaptable).

En las revisiones bibliográficas realizadas por los autores se pudo constatar que el mundo de la internet se inicia con el ARPHANET el cual fue un proyecto de comunicación entre bases militares en Estados Unidos. Los procesos para el envío de datos hacen referencia al modelo TCP/IP, el cual describe todo el proceso que lleva trasladar la información por las 7 capas de comunicación hasta que sea visible en el receptor o el usuario final.

En los años 90, aparece la web 1.0, en la cual se presentaba contenido estático tal como se lo conoce en los niveles formativos de la carrera, en la cual todo el proceso 
para el desarrollo es escrito por un programa y la persona encargada no puede modificar su contenido una vez que se lo ha publicado.

Con el surgimiento de la web 2.0, a diferencia de su predecesora, el contenido de dichas páginas es dinámico, el cual mediante una conexión hacia una base de datos hace que el contenido se muestre según los requerimientos del usuario (Hillar, Gastón C., 2009)

Actualmente se ha denominado la web 3.0, específicamente en esta etapa los entornos son más flexibles y amigables con el usuario, dejándolo que el usuario sea partícipe en el diseño y personalización de su entorno, por lo cual se buscó medios para que el consumidor de los recursos modifique a su gusto el entorno en el cual se encuentra diariamente.

La web 3.0, en su inicio causó polémica debido a que todo el mercado de animación se basaba en un estándar que la compañía Adobe centralizó con el desarrollo de su programa Flash. Las complicaciones con este tipo de animación radicaban en su complejidad y en el tamaño de un archivo en algunos casos era incapaz de mostrarse en ordenadores que poseían un limitado ancho de banda. En estas consideraciones es importante tener en cuenta la metodología de desarrollo.

Roger S. Pressman, Ph.D. (2011) experto Ingeniería del software hace referencia a varias metodologías y herramientas como por ejemplo de modelos:

Modelo de la cascada. - Hay veces en las que los requerimientos para cierto problema se comprenden bien: cuando el trabajo desde la comunicación hasta el despliegue fluye en forma razonablemente lineal. Esta situación se encuentra en ocasiones cuando deben hacerse adaptaciones o mejoras bien definidas a un sistema ya existente (por ejemplo, una adaptación para software de contabilidad que es obligatorio hacer debido a cambios en las regulaciones gubernamentales). También ocurre en cierto número limitado de nuevos esfuerzos de desarrollo, pero sólo cuando los requerimientos están bien definidos y tienen una estabilidad razonable.

Métodos agiles. - Tomando el análisis que hace Kendall citado por Roger S. Pressman, (2011) en relación al ciclo de vida del desarrollo de sistemas, hacen referencia a actividades específicas que se llevan a cabo para desarrollar los sistemas.

Existen varias fases de acuerdo a varios analistas que incluyen:

- Identificación de los problemas, oportunidades y objetivos

- Determinación de los requerimientos humanos de información

- Análisis de las necesidades del sistema

- Diseño del sistema recomendado

- Desarrollo y documentación del software

- Prueba y mantenimiento del sistema

- Implementación y evaluación del sistema

- Prototipos y Herramientas

- Modelado ágil y prototipos

La creación de prototipos de sistemas de información es una técnica valiosa para recopilar rápidamente información específica sobre los requerimientos de información de los usuarios. También indica que la creación de prototipos efectivos se debe llevar 
a cabo en las primeras etapas del SDLC, durante la fase de determinación de requerimientos.

Modelos de proceso incremental. - Hay muchas situaciones en las que los requerimientos iniciales del software están razonablemente bien definidos, pero el alcance general del esfuerzo de desarrollo imposibilita un proceso lineal. Además, tal vez haya una necesidad imperiosa de dar rápidamente cierta funcionalidad limitada de software a los usuarios y aumentarla en las entregas posteriores de software. En tales casos, se elige un modelo de proceso diseñado para producir el software en incrementos.

El modelo incremental combina elementos de los flujos de proceso lineal y paralelo, por lo que aplica secuencias lineales en forma escalonada a medida que avanza el calendario de actividades. Cada secuencia lineal produce "incrementos" de software susceptibles de entregarse de manera parecida a los incrementos producidos en un flujo de proceso evolutivo. Por ejemplo, un software para procesar textos, que se elabore con el paradigma incremental quizá entregue en el primer incremento las funciones básicas de administración de archivos, edición y producción del documento; en el segundo dará herramientas más sofisticadas de edición y producción de documentos; en el tercero habrá separación de palabras y revisión de la ortografía y en el cuarto se proporcionará la capacidad para dar formato avanzado a las páginas.

Debe observarse que el flujo de proceso para cualquier incremento puede incorporar el paradigma del prototipo.

Modelos de proceso evolutivo. - El software, como todos los sistemas complejos, evoluciona en el tiempo. Es frecuente que los requerimientos del negocio y del producto cambien conforme avanza el desarrollo, lo que hace que no sea realista trazar una trayectoria rectilínea hacia el producto final; los plazos apretados del mercado hacen que sea imposible la terminación de un software perfecto, pero debe lanzarse una versión limitada a fin de aliviar la presión de la competencia o del negocio; se comprende bien el conjunto de requerimientos o el producto básico, pero los detalles del producto o extensiones del sistema aún están por definirse. En estas situaciones y otras parecidas se necesita un modelo de proceso diseñado explícitamente para adaptarse a un producto que evoluciona con el tiempo.

Los modelos evolutivos son iterativos. Se caracterizan por la manera en la que permiten desarrollar versiones cada vez más completas del software. En los párrafos que siguen se presentan dos modelos comunes de proceso evolutivo.

PROGRAMACIÓN EXTREMA (XP).- Aunque las primeras actividades con las ideas y los métodos asociados a XP ocurrieron al final de la década de 1980, el trabajo fundamental sobre la materia había sido escrito por Kent Beck citado por Roger S. Pressman, Ph.D. (2011), Una variante de XP llamada XP industrial se propuso en una época más reciente. IXP mejora la XP y tiene como objetivo el proceso ágil para ser usado específicamente en organizaciones grandes.

Valores XP. - Beck define un conjunto de cinco valores que establecen el fundamento para todo trabajo realizado como parte de XP: comunicación, simplicidad, retroalimentación, valentía y respeto. Cada uno de estos valores se usa como un motor para actividades, acciones y tareas específicas de XP.

A fin de lograr la comunicación eficaz entre los ingenieros de software y otros participantes (por ejemplo, para establecer las características y funciones requeridas 
para el software), XP pone el énfasis en la colaboración estrecha pero informal (verbal) entre los clientes y los desarrolladores, en el establecimiento de metáforas para comunicar conceptos importantes, en la retroalimentación continua y en evitar la documentación voluminosa como medio de comunicación.

Para alcanzar la simplicidad, XP restringe a los desarrolladores para que diseñen sólo para las necesidades inmediatas, en lugar de considerar las del futuro. El objetivo es crear un diseño sencillo que se implemente con facilidad en forma de código. Si hay que mejorar el diseño, se rediseñará en un momento posterior.

La retroalimentación se obtiene de tres fuentes: el software implementado, el cliente y otros miembros del equipo de software. Al diseñar e implementar una estrategia de pruebas eficaz por medio de los resultados de las pruebas da retroalimentación al equipo ágil. XP usa la prueba unitaria como su táctica principal de pruebas. A medida que se desarrolla cada clase, el equipo implementa una prueba unitaria para ejecutar cada operación de acuerdo con su funcionalidad especificada. Cuando se entrega un incremento a un cliente, las historias del usuario o casos de uso que se implementan con el incremento se utilizan como base para las pruebas de aceptación. El grado en el que el software diseña la salida, función y comportamiento del caso de uso es una forma de retroalimentación.

Beck, (2004) afirma que la "adhesión estricta a ciertas prácticas de XP requiere valentía". Un término más apropiado sería disciplina más adelante señala que por ejemplo, es frecuente que haya mucha presión para diseñar requerimientos futuros. La mayor parte de equipos de software sucumben a ella y se justifican porque "diseñar para el mañana" ahorrará tiempo y esfuerzo en el largo plazo. Un equipo XP ágil debe tener la disciplina (valentía) para diseñar para hoy y reconocer que los requerimientos futuros tal vez cambien mucho, por lo que demandarán repeticiones sustanciales del diseño y del código implementado.

Al apegarse a cada uno de estos valores, el equipo ágil inculca respeto entre sus miembros, entre otros participantes y los integrantes del equipo, e indirectamente para el software en sí mismo. Conforme logra la entrega exitosa de incrementos de software, el equipo desarrolla más respeto para el proceso XP.

Las herramientas de desarrollo son innumerables bajo las circunstancias en las que se debe transcurrir el proyecto. Antes de poner énfasis en dichas herramientas hay que mencionar que existen como tales dos tipos comúnmente conocidos como leguajes para el desarrollo de aplicaciones, sean estas de escritorio, web o móviles y que son distintas por su entorno y los sistemas operativos, pero cabe recalcar que las empresas hacen todo lo posible para atraer a este tipo de mercado a sus usuarios.

Como tal Visual Basic en es un lenguaje de programación que por ser robusto está enfocado a cualquier variante de programación, sea orientada tanto a objetos como a eventos, pero no son los únicos, podemos describir que el primer lenguaje de uso masivo fue $\mathrm{C}$, y lo representaron con la primera nota musical DO y dicho lenguaje de programación es muy limitado y no soporta el orientarlo a objeto ni a eventos.

Desde sus inicios, el lenguaje $\mathrm{C}$ ha tenido muchas variantes como $\mathrm{C \#}$, el cual fue una adaptación de Microsoft para competir contra el uso frecuente de $\mathrm{C}$, en el cual tuvo una característica principal, el poder crear funciones y objetos para que puedan ser consumidos desde el código, sin afectar a su desempeño, lo cual hizo que $\mathrm{C}$, quedara un poco en el olvido y además de la mezcla de estos dos lenguajes surgió Java y su adaptación para web Javascript. 
Como el autor menciona con anterioridad, las grandes compañías dedicadas al desarrollo de software han creado sus propios lenguajes y sistemas operativos, pero actualmente el software libre a dado un salto muy importante en el uso cotidiano, hoy en día se encuentran más páginas web y programas de escritorio creados en lenguajes libres que los propietarios.

El término software libre en algunos casos es tomado solo porque es gratuito, debemos tener en cuenta que para que un programa sea considerado como software libre debe tener tres aspectos fundamentales.

1. Debe estar disponible en todo momento.

2. El código debe ser abierto, si en caso se desea modificar.

3. No debe tener ningún costo.

Como tal las herramientas se basan en los lenguajes de programación para el desarrollo de páginas web, de tal manera que un Lenguajes de Programación Libre es aquel que cumple con estos principios. De otra manera, en los Software Propietario el código no se encuentra disponible para realizar modificaciones así, este no tenga un valor comercial.

Como indica el autor Álvarez, M. A. (2001) en su portal desarrollo web.com, PHP, es un lenguaje de programación del lado del servidor gratuito e independiente de plataforma, rápido, con una gran librería de funciones y mucha documentación.

Un lenguaje del lado del servidor es aquel que se ejecuta en el servidor web, justo antes de que se envíe la página a través de Internet al cliente. Las páginas que se ejecutan en el servidor pueden realizar accesos a bases de datos, conexiones en red, y otras tareas para crear la página final que verá el cliente. El cliente solamente recibe una página con el código HTML resultante de la ejecución de PHP. PHP es otro lenguaje de programación enfocado hacia páginas web, que no se compila y se muestra de manera tal como fue escrito, el cual se enmarca dentro del llamado Software Libre.

Visual Studio es un aglomerado de aplicaciones creadas por Microsoft para ofrecer a los desarrolladores un entorno de desarrollo de peso para las plataformas Windows y .NET. Visual Studio se puede utilizar para escribir aplicaciones de consola, aplicaciones de Windows, servicios de Windows, aplicaciones de Windows Mobile, las aplicaciones ASP.NET y servicios web ASP.NET, en la elección de C ++, C \#, VB.NET, J \#, y más. Visual Studio también incluye varias herramientas de desarrollo adicionales, tales como Visual SourceSafe; que se incluyen herramientas que dependen en gran medida de la edición de Visual Studio que está utilizando.

Microsoft tiene una larga historia con las herramientas de desarrollo y Visual Studio es la culminación natural de estos esfuerzos. Desde hace varios años, Microsoft envía herramientas de desarrollo individual como Visual $\mathrm{C}++$ y Visual Basic, pero a partir de 1997 se comenzó a ofrecer Visual Studio, que combina todos estos ambientes en una sola aplicación.

Ha habido numerosas versiones de Visual Studio desde su inicio. Visual Studio 6 coincidió con el lanzamiento de Visual Basic 6; Visual Studio.NET 2002 fue puesto en libertad junto con la versión 1.0 de .NET Framework, y se revisó de nuevo con la versión 1.1 de .NET Framework, momento en el que fue bautizado como Visual Studio .NET 2003. La siguiente versión de Visual Studio, fue llamada Visual Studio 2005 y en la actualidad se cuenta con la versión beta del Visual Studio 2015. 


\subsection{Fundamentos acerca de la competencia desarrollar sistemas web.}

La expresión "competencias" tiene diversos usos sociales, algunos más especializados que otros. Así se usa en la vida corriente, en la economía, en el derecho, en los deportes, en el mundo de trabajo, entre otras. Aunque el estudio del término no es objeto de este trabajo, si resulta oportuno señalar su importancia. Es necesario captar esos usos, acentos y dispersiones que el vocablo tiene, disciplinar y cotidianamente. La importancia reside en que, por ejemplo, se podría comprender las competencias exclusivamente desde el punto de vista económico y entonces, se pensaría en formar profesionales para que compitan en el mercado laboral, asunto importante, pero no el único en que el que ha de reparar la pedagogía como discurso y práctica que se propone finalmente el desarrollo humano integral. ¿Qué entender por competencias desde el discurso específicamente pedagógico? (Fuentes, 2002)

El concepto de competencia constituye uno de los más tratados y polémicos en las ciencias pedagógicas actuales; también va siendo uno de los más citados y trabajados en este tipo de investigaciones actualmente. Este vocablo ha sido usado teniendo en cuenta diferentes significados. Para González, et al (2003), competencia "tiene que ver con una combinación integrada de conocimientos, habilidades y actitudes conducentes a un desempeño adecuado y oportuno en diversos contextos. La flexibilidad y capacidad de adaptación resultan claves para el nuevo tipo de logro que busca el trabajo y la educación como desarrollo general, para que las personas hagan algo con lo que saben" (González, D.C., 2003:4).

El término competencia se define, entonces, como un "saber hacer, sobre algo, con de terminadas actitudes", Es decir, como una medida de lo que una persona puede hacer bien como resultado de la integración de sus conocimientos, habilidades, actitudes y cualidades personales. La definición anterior destaca tres aspectos esenciales del término competencia: carácter práctico, contenido que se sabe hacer y la actitud que asume en su actuación. O sea, se hace referencia a los aprendizajes a través de los cuales un individuo se hace competente: "sabe quién es, sabe hacer, emprender y hacerlo con otros y, finalmente, cómo hizo para saberlo." (González, D. C. et al, 2003, p.4).

Por su parte, (Martínez Carretero, 2005, p. 20) define la competencia como: "una compleja estructura de atributos necesarios para el desempeño de situaciones específicas. Es una compleja combinación de atributos (conocimiento, actitudes, valores y habilidades) y las tareas que se tienen que desempeñar en determinadas situaciones". Este concepto es bastante cercano al asumido por el autor anterior, por considerar que es fundamental en él la inclusión de los términos: conocimientos, actitudes, valores y habilidades en función de las tareas que propician la materialización de los mismos, así como la presencia de un modo de actuación profesional, sobre todo, cuando se trata del desarrollo de competencias asociadas al perfil profesional de un futuro egresado, como es el caso de la educación superior.

López Calichs enfoca a las competencias como la expresión de un conjunto de atributos de la persona que van más allá del conocimiento y abarca la formación de manera más integral, incluyendo las habilidades, actitudes, comunicación y personalidad, y por otra, la relación entre el conjunto de dichos atributos y el resultado o desempeño, lo que compromete a su vez, la actualización y perfeccionamiento constante del conocimiento y de las formas de hacer. La competencia, como un enfoque integral de formación desde su diseño mismo, conecta el mundo del trabajo y la sociedad en general, con el mundo de la educación. (López E., 2005) 
Por su parte Cejas Yanes, define que la competencia es un sistema de componentes (cognitivos, metacognitivos, motivacionales y cualidades de la personalidad) que posee un individuo para desenvolverse eficientemente en su vida como ser social en todas las facetas. (Cejas, 2006)

A partir de las definiciones anteriores se puede constatar en el concepto de competencia no solo están presentes los conocimientos y habilidades en una actividad sino también se refiere de alguna manera a los modos de actuación del individuo en el entorno en el que lleva a cabo su función social.

Por lo tanto puede observarse en las definiciones anteriores que las competencias, pueden ser definidas como aprendizajes o logros complejos que integran aspectos cognitivos, procedimentales, actitudinales, habilidades, características de la personalidad y valores, que puestos en práctica en un determinado contexto, tendrán un impacto positivo en los resultados de la actividad desempeñada (Feo Mora, 2010, p. 227) Las competencias posibilitan que el estudiante consiga de manera gradual niveles superiores de desempeño no sólo técnicos, operativos y manuales requeridos en el área tecnológica, sino también aquellas que resultan esenciales para la vida, para la comunicación humana, las que permiten la convivencia, el desarrollo personal.

En este sentido, hablar de competencias implica el desarrollo de conocimientos, habilidades, destrezas y actitudes desde un contexto real que posibilite el aprendizaje significativo, crítico y reflexivo, así como un compromiso proactivo de participación y liderazgo que requiere ingenio y creatividad para enfrentar los retos de la calidad educativa (Pérez, 2013).

Para otro autor (D’Angelo Hernández, 2015) las competencias son estructuras psicológicas integrativas, de nivel intermedio, que complementan o articulan las funciones de las estructuras principales de la personalidad ante situaciones que demandan un desempeño determinado como expresión del comportamiento de la persona en su contexto social y en un ambiente específico de acción.

Por otro lado "La competencia no reside en las capacidades que un educando posee, sino en su movilización para resolver problemas. "Saber", además no es poseer sino utilizar, ya que al poner en práctica una acción es que se llega a ser competente. Por otra parte, "saber hacer" no es aplicar rutinariamente los saberes por el educando, sino es un "saber actuar". El "saber actuar" conlleva un conjunto de acciones, donde la ejecución de cada uno es dependiente del cumplimiento del todo. La competencia exige saber encadenar y desencadenar diferentes actuaciones y no solo aplicarlas aislada y mecánicamente."(Ledo, Perea, Oliva, \& Meriño, 2016, p. 30)

Otros autores asumen las competencias como “... un conjunto de conocimientos, habilidades, valores, cualidades y comportamientos de la personalidad que se movilizan en función de las necesidades individuales y sociales, permitiéndose el desempeño satisfactorio en el ejercicio de la profesión, teniéndose en cuenta que una vez adquiridas, cambian y se desarrollan constantemente y estas no pueden explicarse, demostrarse y evaluarse independientemente del contexto y sus exigencias(Véliz Martínez, Jorna Calixto, \& Berra Socarrás, 2016)

Para varios autores (Castañeda, 2017; Corral \& Fernández, 2017; Ledo et al., 2016; Mozo, Ramírez, \& Téllez, 2016) las competencias pueden ser definidas como aprendizajes o logros complejos que integran aspectos cognitivos, procedimentales, actitudinales, habilidades, características de la personalidad y valores, que puestos en 
práctica en un determinado contexto, tendrán un impacto positivo en los resultados de la actividad desempeñada.

Por lo que se concluye que la formación basada en competencias supone, por tanto, un conjunto de conocimientos, habilidades, actitudes, aptitudes, motivaciones y valores, que posee el individuo para su desempeño óptimo en una ocupación o función productiva determinada. Reconocer el concepto de competencia en el ámbito educacional, significa tener la posibilidad de incorporar al proceso docente educativo una nueva concepción, dirigida a la formación de un profesional con aptitudes y actitudes para que responda a las exigencias que la sociedad le plantea, no sólo en el marco de la profesión sino en lo social, donde de forma consecuente sea un seguidor fiel de su historia y tradiciones, reflexivo y sobre todo capaz de enfrentarse y atemperarse a los nuevos retos y exigencias de la ciencia y la técnica para transformar el medio, desarrollarlo y con ello transformarse a sí mismo.

Siguiendo con estos autores as competencias van a ser adquiridas a lo largo de todo el proceso formativo, y no pueden entenderse fuera del contexto particular donde se ponen en juego, o sea, no pueden separarse de las condiciones específicas del escenario docente donde se evidencian y de los procesos formativos previstos. Por ello es que las competencias van más allá de la mera especialización técnica, para incluir también las dimensiones relacionales y sociopolíticas. El comportamiento técnico y metodológico ha de completarse con el comportamiento personal, político y social.

Para otros autores (Reyes Pérez, Rodríguez Fernández, \& Cruz Rodríguez, 2015, p. 925) “... es integrar y movilizar sistemas de conocimientos, habilidades, hábitos, actitudes y valores para la solución exitosa de aquellas actividades vinculadas a la satisfacción de sus necesidades cognitivas y profesionales, demostradas en su desempeño, al tomar soluciones y corregir las situaciones que se presenten en su esfera de trabajo, su contexto".

De esta manera se considera por el autor (Fernández \& Bueno, 2016, p. 20) “... la competencia es un proceso más que un estado; es poniendo en práctica-acción la competencia como se llega a ser competente. En este sentido de actuación profesional, también podemos aludir a que la competencia está contaminada de ética (con uno mismo, con los demás, con la comunidad...). Es necesario desarrollar una base ética en la actuación de los profesionales, teniendo en cuenta su ser y el de los demás, así como el entorno ambiental. En este último punto incluso podemos hablar del componente ecológico de la competencia (saber ser y saber estar en contexto)"

A pesar de la diversidad de connotaciones que tienen las definiciones de competencia, analizadas en la literatura (Feo Mora, 2010; Ledo et al., 2016; Mozo et al., 2016; Véliz Martínez et al., 2016) es posible distinguir algunos rasgos característicos:

1. Se combinan en forma de sistema los conocimientos, procedimientos, actitudes y valores que el individuo ha de saber, saber hacer, saber ser y saber ser para saber actuar en la solución de problemas, tareas, funciones y responsabilidades de su práctica profesional.

2. Las competencias sólo pueden ser definidas y obtenidas en relación a la actividad, mediante un desempeño profesional especifico en un contexto determinado. 
3. El contexto es un elemento clave, que expresa las potencialidades de la persona para orientar su actuación en el ejercicio de la profesión con iniciativa, flexibilidad y autonomía, en escenarios heterogéneos y diversos.

4. Se evalúa mediante el rendimiento laboral obtenido sobre la base de criterios acordados e implica un compromiso individual, institucional, educacional y social.

5. Se basan en un determinado desarrollo económico, cultural, histórico, social, en la formación humanista, el desarrollo tecnológico, así como en un posicionamiento político e ideológico.

Además, estos autores ya señalados (Feo Mora, 2010; Ledo et al., 2016; Mozo et al., 2016; Véliz Martínez et al., 2016) refieren tres grupos generales de competencias:

- Competencias básicas. Son aquellas en las que la persona construye las bases de su aprendizaje (interpretar y comunicar información, razonar creativamente y solucionar problemas, entre otras). Abarcan la capacidad de "aprender a aprender". Pone en movimiento diversos rasgos cognitivos, como la capacidad de situar y comprender de manera crítica, las imágenes y los datos que le llegan de fuentes múltiples; la aptitud para observar, la voluntad de experimentación y la capacidad de tener criterio y tomar decisiones. Entre las competencias básicas que suelen incluirse en los currículos se encuentran la comunicación verbal y escrita, la lectura y la escritura, las nociones de aritmética, el trabajo en equipo, la resolución de problemas y la enseñanza de lenguas extranjeras.

- Competencias personales. Son aquellas que permiten realizar con éxito las diferentes funciones en la vida (actuar responsablemente, mostrar deseo de superación y aceptar el cambio, entre otras). Están en función de las capacidades y potencialidades de expresión de un grupo de características que se manifiestan en dependencia del ambiente en que se desarrolle la actividad, tales como seguridad en sí mismo, capacidad para dominar los sentimientos y las tensiones emocionales, curiosidad, argumentación crítica y capacidad analítica.

- Competencias profesionales. Son las que garantizan cumplir con las tareas y responsabilidades de su ejercicio profesional. Las cualidades de las personas para desempeñarse productivamente en una situación de trabajo, no sólo dependen de las situaciones de aprendizaje escolar formal, sino también del aprendizaje derivado de la experiencia en situaciones concretas de trabajo.

En relación con las competencias profesionales el autor se adscribe a la clasificación dada por (Feo Mora, 2010; Ledo et al., 2016; Mozo et al., 2016; Véliz Martínez et al., 2016) a partir de su relación con la presente investigación, concerniente al estudio de las competencias profesionales.

$\varnothing \quad$ Competencia básica: son aquellas en las que la persona construye las bases de su aprendizaje, constituyen configuraciones de la personalidad del sujeto y expresión en sus características individuales, le permiten adaptarse a los diferentes contextos, tanto laborales como de otra índole.

$\varnothing \quad$ Competencia personal: son aquellas que permiten realizar con éxito las diferentes funciones en la vida, actuar responsablemente, mostrar deseo de superación, aceptar el cambio, autoestima realista, principios propios, responsabilidad y toma de decisiones. 
$\varnothing \quad$ Competencias profesionales: las competencias profesionales están integrada por conocimientos, habilidades, actitudes, valores motivos, aptitudes y capacidades que debe poseer el individuo para su desempeño en su profesión.

El autor considera que las competencias profesionales son más que la sumatoria de atributos anteriormente expuestos, se trata de poner en práctica conocimientos disciplinares, conceptos-argumentaciones-teorías, criterios procedimentales e instrumentales que permiten desarrollar correctamente la actividad laboral en base de acciones razonadas y sistemáticas que viabilizan la contribución profesional con niveles de complejidad, autonomía y variedad (competencia genérica), demostrar la idoneidad en el puesto de trabajo (competencia laboral), la construcción de aprendizajes y la adaptación a los diferentes contextos (competencia básica).

Para otros autores las competencias profesionales son "...una integración de saberes: los conocimientos <saber>, las habilidades <saber hacer> y las actitudes y valores que implican un <saber ser, saber estar y saber por qué se hace> que se adquieren durante la formación, donde el aprendizaje está estrechamente ligado a la práctica, que como actividad transformadora, crea valores, que se expresan a través de un desempeño, que en cumplimiento de los principios de la ética y el profesionalismo debe ser de calidad" (Ortiz García, Vicedo Tomey, \& García Capote, 2016, p. 14). De modo que puede considerarse a la ética, no como una competencia aislada, sino que debe ser un componente fundamental de la actuación profesional representado en cada una de las competencias. Para estos autores la competencia se expresa en la acción, por lo que se considera en esta investigación que la competencia no reside en los recursos que la persona posee sino en la movilización misma de los recursos. Para ser competente es necesario poner en juego el repertorio de recursos: a) los conocimientos aptitudes y destrezas técnicas (saber), b) las formas metodológicas de proceder en el trabajo (saber hacer), c) las pautas y formas de comportamiento individuales y colectivas (saber ser),d) las formas de organización e interacción (saber ser).

De la misma manera en la literatura consultada (Galán, Ramírez, \& Pacheco, 2014; Ledo et al., 2016; Martínez, 2016; Pérez, 2013; Sabirón \& Arraiz, 2016) se considera que, en resumen, las competencias profesionales se han convertido en un instrumento para el diálogo y la negociación entre la educación y el trabajo, ya que fortalecen la necesidad de que todo proyecto curricular sea pertinente en relación con el encargo social, y que su perfil profesional esté en correspondencia con el desempeño profesional, de forma que conduzca a que los individuos encuentren en ella una formación propiciadora para su desarrollo integral y que se traduzca en una posibilidad real de incorporación a la sociedad contemporánea donde viven. Una vez analizado la competencia profesional y asumida esta última definición es importante esclarecer la estructura de la competencia profesional desarrollar sistemas web.

Todas las concepciones analizadas sobre las competencias y específicamente las profesionales, permiten aseverar que el desarrollo de sistemas web es una competencia del ingeniero informático. Es por ello que se considera necesario para continuar el análisis de la referida competencia abordar las habilidades que la componen. Se pueden enunciar cinco habilidades generalizadoras esenciales de un profesional informático relacionada el desarrollo de aplicaciones web. las cuales se han denominado de la siguiente forma:

- Gestionar el proyecto. La gestión del proyecto web pasa por determinar la metodología de desarrollo del proyecto, determinar los riesgos del proyecto y determinar los costos, tiempo y esfuerzo que lleva el desarrollo del proyecto. 
- Describir los procesos funcionales del sistema que se representan, el sistema de acciones a realizar llevaría dos acciones, una primera encaminada a establecer los requisitos funcionales y no funcionales del sistema y otra acción encaminada a elaborar el modelo de análisis que se va a realizar utilizando el sistema de artefactos adecuados para la metodología declarada en la gestión del proyecto que le permite obtener el modelo de análisis del sistema que se pretende desarrollar.

Modelar la arquitectura del sistema en la cual se establecen dos acciones fundamentales, la primera modelar la estructura del sistema a partir de los artefactos declarados en la metodología y la segunda modelar las relaciones entre los componentes.

- Implementación del sistema web. En el análisis de esta habilidad se asumen los criterios sobre la implementación como actividad informática. Se pueden enunciar cuatro acciones esenciales de un profesional informático relacionada con la implementación. La primera se denomina en interpretar los procesos y estructuras que se representan en el modelo utilizando el sistema de símbolos adecuados que le permite estructurar las representaciones que se han analizado hasta el momento. La segunda se denomina comprender los modelos realizados por otros en los procesos de informatización y llevarlos a cabo según la concepción de los encargados de estos procesos: analistas y diseñadores. Para ello es importante retomar el proyecto, ahora como eje articulador de los procesos formativos en los ingenieros informáticos. La tercera acción está relacionada con la selección de los lenguajes, herramientas y tecnologías más adecuadas para la concreción del modelo en la solución de la problemática planteada al proyecto. Dentro de esta tercera acción, la selección de los frameworks, ides y cms, en caso que el proyecto los necesite, es importante para la implementación de los sistemas. Y la última y no menos importante está relacionada con la elaboración de estrategias de concreción de estos procesos en la práctica. La integración de estas cuatro acciones en un sistema armónico conjuntamente con los conocimientos acerca de la implementación permitirá a los estudiantes conformar con éxito una implementación del sistema.

- Depuración de errores en un sistema. La necesaria diferenciación de los procesos de depuración que cada estudiante realiza y si se estructura de manera individual posibilita la expresión de sus experiencias, conocimientos y habilidades relacionadas con este proceso de llevar a vías de hecho los modelos realizados. Ello les permite integrar sus proyectos con el resto e ir aprendiendo de los demás colegas. Teniendo en cuenta estos elementos, es que se aprecia en esta tesis que la depuración es una habilidad generalizadora en el ingeniero informático y a continuación se analizará su estructura.

De la misma manera que las anteriores se analizará la depuración como una habilidad generalizadora del ingeniero informático. Es válido aclarar que en la depuración se distinguen dos grupos de acciones: pruebas y la corrección de los errores detectados, la depuración. Se pueden enunciar cinco acciones esenciales de un profesional informático relacionada con la depuración. Una primera se denomina elaborar plan de pruebas que se representan el sistema de pruebas que se van a realizar utilizando el sistema de artefactos adecuados que le permite estructurar las pruebas que se han analizado hasta el momento. La segunda se denomina elaborar la estrategia de pruebas en los procesos de informatización y llevarlos a cabo según la concepción de los encargados de estos procesos: gestor de pruebas y probador. Para ello es importante retomar el proyecto, ahora como eje articulador de los procesos formativos en los ingenieros informáticos. La tercera acción está relacionada con la ejecución de las pruebas para la concreción del plan y la estrategia de pruebas en la solución de la 
problemática planteada al proyecto. Dentro de esta tercera acción, la selección de las herramientas automáticas, en caso que el proyecto los necesite, es importante para la depuración de los sistemas por variadas causas establecidas en la literatura (Bobilloa \& Stracciab, 2016; Lima, Lima, Monteiro, Júnior, \& Gomes, 2012; Ruiz, 2011; Silió, 2005). La cuarta acción está relacionada con la selección del método de depuración de estos procesos en la práctica. La última, y no menos importante, es la ejecución de la depuración de los errores detectados en el proceso de informatización de las organizaciones. La integración de estas cinco acciones en un sistema armónico conjuntamente con los conocimientos acerca de la depuración permitirá a los estudiantes conformar con éxito un proyecto.

La formación basada en proyectos permite al estudiante aplicar los contenidos apropiados y analizar rápidamente la pertinencia de éstos para su formación profesional. En un entorno de proyecto el estudiante desempeña los roles de su futuro profesional y va articulando los modos de actuación que desempeñará en el futuro, de tal manera que se va articulando dentro de su proyecto de vida la futura profesión que va a desempeñar. Este proceso tiene una especial relevancia en la juventud por ser una de las características fundamentales de la situación social de desarrollo en la cual se encuentran.

Sin embargo, la educación de los valores en el proyecto es también importante a tener en cuenta en este artículo. En este mismo orden de ideas, se van conformando aquellos valores propios de la profesión como la responsabilidad, honestidad y humildad (de Castro \& de Sá, 2002). La responsabilidad es uno de los valores más importantes en el profesional informático por las características propias de la actividad, así como por el impacto social que tienen las tecnologías para la sociedad. En cualquiera de los roles que deba desempeñar debe ser responsable de sus actos y constituir un verdadero eje regulador de sus acciones puesto que de ellas depende las del resto del proyecto. El desarrollo de sistemas web es una de las actividades con potencial para el desarrollo de la responsabilidad por el papel que desempeña en la concreción del proceso de informatización a desarrollar y por su marcado carácter práctico. Además, es esencial el proceso de desarrollo para que el proceso de informatización carezca de errores y sea eficiente.

Durante el desarrollo del proyecto la honestidad con los colegas en el proceso de desarrollo es primordial para entender las relaciones sociales que se establecen. Estas relaciones en las cuales el proyecto, el posicionamiento de la empresa y la confianza entre los integrantes tienen un papel fundamental.

La educación de estos valores debe basarse en el conjunto de vivencias que hacen comprender al sujeto su responsabilidad ante los demás integrantes del proyecto y la sociedad por el resultado del proyecto que van a obtener. Este proceso debe estar centrado en la importancia del rol que desempeña y el resultado de la actividad para la organización. En un ambiente de proyecto todos los roles son importantes. En estos contextos es esencial la explicación detallada de los errores, así como la oportunidad de expresarlos y corregirlos sin constituir una oportunidad de castigo, a través del diálogo, la confrontación y la polémica constante y constructiva. También es importante para el desarrollo de sistemas ir asignando tareas de mayor complejidad y que involucren con el resto de los integrantes del proyecto para explicar las especificidades de su proyecto en la organización.

La integración de estos valores, en el accionar del estudiante que se estructuran en la actividad informática, con el proyecto de vida hace que se incorporen de manera real 
al potencial regulador de la personalidad del profesional. De esta manera estos valores se constituirán en parte de la subjetividad del sujeto y no generarán formalismos.

La integración de estudiante - realidad - enseñanza propicia que el trabajo de los estudiantes adquiera un carácter social tanto por la implicación de los resultados del proyecto para las organizaciones, así como el sistema de relaciones a desarrollar con el resto del colectivo en la solución de los problemas. Lo anteriormente planteado conlleva al análisis de la situación y una postura reflexiva ante las críticas y los cuestionamientos.

La transformación de la realidad por parte del estudiante a partir del proceso de informatización y la selección de las metodologías, herramientas y lenguajes necesarios para lograrlo evidencian el carácter activo de la función reguladora de su personalidad. La amplia variedad de herramientas para una misma actividad, así como la selección de las metodologías implica que se han tomado decisiones con respecto al proyecto y los modelos que en él intervienen. Este proceso de transformación debe ocurrir primeramente en el plano mental siendo importante de la imaginación, con lo que se contribuye a su desarrollo. Este es uno de los aspectos esenciales que diferencian la actividad de desarrollo de otras actividades informáticas. En este caso, por la complejidad en el plano mental y práctico de las acciones que intervienen en un proceso de desarrollo web, los estudiantes aprecian con mayor claridad cómo se da el tránsito de lo abstracto a lo concreto durante la actividad informática. De esta manera los estudiantes van integrando estas vivencias de participación en actividades que intervienen en este proceso entre otras actividades al mismo tiempo que van regulando el aprendizaje de la depuración en la actividad informática.

A partir de la búsqueda de problemas en la realidad se comienza el ciclo de vida de un software hasta que concluye con la puesta punto y mantenimiento. Los problemas derivados del proyecto individual motivan a los estudiantes hacia su solución y en ellos se encuentran los conocimientos del curso que a su vez generan las situaciones problémicas para los demás estudiantes. Las acciones y operaciones asociadas al desarrollo web, a partir de la utilización del conjunto de símbolos y signos asociados a ello, y el trabajo conjunto con el resto de colegas de mayor experiencia van estructurando diferentes escenarios de su futuro perfil profesional.

En cada encuentro, a partir de la interacción previa entre estudiante - profesor grupo, se determinan las situaciones problémicas para los restantes estudiantes. Es el profesor quien decide la situación problémica a presentarse en el encuentro basándose previamente en la interacción grupal y el desarrollo de los proyectos de los estudiantes. Para muchos autores (de Castro \& de Sá, 2002; Gutiérrez Alea, 2012; Jiménez, Barrera Jiménez, \& Hernández Amaro, 2015; López Valero, 2010; Mulder, Lemmen, \& van Veen, 2002) la formación de habilidades en la informática se da en el momento de enseñar un sistema en particular. En este artículo se asume una concepción sistémica en la cual se aborda el proceso como un todo integrado, en el cual se le presta especial atención a la concatenación de los conceptos y procedimientos informáticos que no se pueden formar en una única clase como en el caso que ocupa este artículo: la competencia depurar procesos de informatización a organizaciones. Por el carácter interdisciplinar y la complejidad de su estructura, su formación en el profesional informático debe abordarse desde una postura sistémica.

Una vez develada la estructura de la competencia es posible entonces definir la competencia desarrollo de sistemas web como una integración de recursos cognitivos, afectivos, volitivos y autorreguladores relacionados con el desarrollo de los sistemas web que se adquieren durante la formación como profesional informático 
estrechamente ligado a la práctica en forma de proyectos, que se expresan a través de su desempeño profesional con calidad a partir de la actividad transformadora de las organizaciones en las cuales se encuentran

Para este artículo la competencia desarrollar sistemas web es una de las más sistémicas e integradoras de la actividad profesional informática. Constituye un eje central en dos roles de la actividad informática: gestor de pruebas, analista, programador, probador y gestor de proyecto. Además, juega un papel esencial como concreción de los modelos obtenidos para las acciones de informatización de procesos. Por ende, la formación de competencia comienza en el segundo año de la carrera y culmina cuando el estudiante expresa su proceso de investigación en forma de memoria escrita en el cual integra todos los modelos estudiados en la carrera para describir el proyecto y los implementa. Ya el proceso de desarrollo de esta competencia corresponde a su ámbito laboral a partir de las diversas problemáticas que este profesional debe resolver en el ámbito organizacional.

\section{Conclusiones}

El desarrollo de sistemas web es una actividad necesaria en la formación de los profesionales informáticos por el impacto que éstas tienen en la sociedad actual. Para ello existen muchas metodologías y procesos que un profesional informático puede seleccionar para su proyecto. De la misma manera el nivel de integración de los conocimientos que necesita para solucionar un proyecto de este tipo es bastante complejo.

El análisis de la competencia desde un enfoque culturalista lleva a profundizar en su esencia como sistema de componentes y no asociada al mercado laboral. La competencia desarrollar sistemas web ha sido definida a partir del análisis de un conjunto de definiciones de competencias y sobre la base de considerarla como una competencia profesional.

\section{Referencias}

Castañeda, HAA (2017). Estado actual de las competencias TIC de docentes. Puente, 9(2), 23-32.

Corral, ON y Fernández, JF (2017). El profesional de la museología social. Competencias, habilidades y futuro para su implicación en el desarrollo territorial. e-rph-Revista electrónica de Patrimonio Histórico, 19, 152-173.

de Castro, EB, \& de Sá, MAD (2002). Habilidades, Competências, Valores e Atitudes-Um Perfil Para o Profissional de Computação e Informática. Paper presented at the Anais do Congresso da Sociedade Brasileira de Computação, Florianópolis, Brasil.

Dey, PP, Khan, M, Amin, M, Sinha, BR, Badkoobehi, H, \& Jawad, S (2016). Managing interacting software project risks. Pressacademia, 2(1), 427-427.

Feo Mora, R (2010). Orientaciones básicas para el diseño de estrategias didácticas. Tendencias pedagógicas, 16, 221236.

Fernández, JT y Bueno, CR (2016). Evaluación de competencias profesionales en Educación Superior: retos e implicaciones. Educación XX1, 19(1), 17 - 38.

Galán, YJ., Ramírez, MG y Pacheco, JO (2014). Evaluación por competencias. Entre la tradición y el cambio. Revista Iberoamericana de Producción Académica y Gestión Educativa, 2, 1 - 21.

Grillo, M., Cardinale, Y., \& Aguilar, J. (2015). CrESTa: Ejecución Tolerante a Fallas de Servicios Web Compuestos basado en Crónicas. Selección, 2(1), 1 - 13. 
Gutiérrez Alea, M. (2012). Una metodología para contribuir al desarrollo de la habilidad resolver problemas en la disciplina Lenguajes y Técnicas de Programación, en estudiantes de la carrera de Licenciatura en Educación, especialidad de Informática. (Doctor en Ciencias Pedagógicas), Universidad Pedaógica Enrique José Varona, Academia de Ciencias de Cuba.

Jain, R., \& Suman, U. (2015). A Systematic Literature Review on Global Software Development Life Cycle. ACM SIGSOFT Software Engineering Notes, 40(2), 1-14.

Jiménez, R. B., Barrera Jiménez, A. D., \& Hernández Amaro, L. E. (2015). Algunas consideraciones en torno al desarrollo de habilidades profesionales del ingeniero informático y el rol de la comprensión de texto en la modelación de algoritmos computacionales. Revista Científico Metodológica Mendive, 50.

Ledo, M. J. V., Perea, R. S. S., Oliva, B. F. y Meriño, A. L. G. (2016). Educación basada en competencias. Educación Médica Superior, 30(1), 23 - 45.

Lima, M. M. d., Lima, A. R. d., Monteiro, A. C. C., Júnior, E. H. C y Gomes, L. d. Q. L. (2012). Uma Revisão Sistemática da Literatura dos Processos de Desenvolvimento de Software Educativo. Paper presented at the Simpósio Brasileiro de Informática na Educação Rio de Janeiro.

López Valero, A. E. F., Eduardo (2010). El aprendizaje intencional y los entornos informatizados, medios para el desarrollo de las habilidades metalingüísticas: un paso hacia adelante en el área de didáctica de la lengua y la literatura. . In U. d. Murcia (Ed.), Enseñanza e Informática (Vol. 4). España: Editorial de la Universidad de Murcia.

Martínez Carretero, J. M. (2005). Los métodos de evaluación de la competencia profesional: la evaluación clínica objetivo estructurada (ECOE). Educación Médica, 8, 18-22.

Martínez, F. M., \& Carmona, G. . (2016). Aproximación al concepto de "competencias emprendedoras": Valor social e implicaciones educativas. . REICE. Revista Iberoamericana sobre Calidad, Eficacia y Cambio en Educación, 7(3), 83 98.

Mozo, D. B., Ramírez, E. F. M., \& Téllez, K. A. P. (2016). Las competencias profesionales: un enfoque de formación y desarrollo de la expresión escrita en las universidades médicas. . Humanidades Médicas, 16(3), 519 - 531.

Mulder, F., Lemmen, K., \& van Veen, M. (2002). Variety in views of university curriculum schemes for informatics / computing / ICT A comparative assessment of ICF-2000 / CC2001/ Career Space. Paper presented at the ICTEM 2002 Florianopolis.

Pérez, MCSN. (2013). El docente en el enfoque por competencias. Pensamiento. Papeles de filosofía, 1(1), 177-186.

Pressman, RS (2011). Ingeniería del software: Un Enfoque Práctico (Séptima Edición ed.). New York, USA: McGRAWHILL Higher Education.

Pressman, RS, \& Lowe, D. (2013). Web Engineering: A Practitioner's Approach: McGraw-Hill Higher Education.

Reyes Pérez, AD., Rodríguez Fernández, M., \& Cruz Rodríguez, J. (2015). Necesidad del enfoque por competencias en la formación profesional en cirugía endoscópica. Medisur, 13(6), 922 - 926.

Ruiz, K. (2011). Métricas para la evaluación de la calidad de un objeto de aprendizaje. . (Tesis en opción al Título de Ingeniero Informático), Universidad de Matanzas, Matanzas, Cuba.

Sabirón, F., \& Arraiz, A. (2016). Aprendiendo de la evaluación: decálogo para la evaluación auténtica de competencias profesionales a través del portafolio. Revista Iberoamericana de Evaluación Educativa, 6(1), 135-152.

Sudhaman, P., \& Thangavel, C. (2015). Efficiency analysis of ERP projects-software quality perspective. International Journal of Project Management, 33(4), 961-970.

Williams, B., \& Figueiredo, J. (2014). From Academia to Start-up: A Case Study with Implications for Engineering Education. International Journal of Engineering Pedagogy, 4, 24-31. 